\title{
A systematic review of cognitive decline in dementia with Lewy bodies versus Alzheimer's disease
}

\author{
Monica H Breitve ${ }^{1,2^{*}}$, Luiza J Chwiszczuk ${ }^{1,3}$, Minna J Hynninen ${ }^{4,5}$, Arvid Rongve ${ }^{1,2,6}$, Kolbjørn Brønnick ${ }^{7,8}$, \\ Carmen Janvin ${ }^{9}$ and Dag Aarsland ${ }^{9,10}$
}

\begin{abstract}
Introduction: The aim of this review was to investigate whether there is a faster cognitive decline in dementia with Lewy bodies (DLB) than in Alzheimer's disease (AD) over time.

Methods: PsycINFO and Medline were searched from 1946 to February 2013. A quality rating from 1 to 15 (best) was applied to the included studies. A quantitative meta-analysis was done on studies with mini mental state examination (MMSE) as the outcome measure.

Results: A total of 18 studies were included. Of these, six (36\%) reported significant differences in the rate of cognitive decline. Three studies reported a faster cognitive decline on MMSE in patients with mixed DLB and AD compared to pure forms, whereas two studies reported a faster decline on delayed recall and recognition in AD and one in DLB on verbal fluency. Mean quality scores for studies that did or did not differ were not significantly different. Six studies reported MMSE scores and were included in the meta-analysis, which showed no significant difference in annual decline on MMSE between DLB (mean 3.4) and AD (mean 3.3).
\end{abstract}

Conclusions: Our findings do not support the hypothesis of a faster rate of cognitive decline in DLB compared to AD. Future studies should apply recent diagnostic criteria, as well as extensive diagnostic evaluation and ideally autopsy diagnosis. Studies with large enough samples, detailed cognitive tests, at least two years follow up and multivariate statistical analysis are also needed.

\section{Introduction}

Dementia with Lewy bodies (DLB) and Alzheimer's disease $(\mathrm{AD})$ are the two most common subtypes of neurodegenerative dementia, representing 15 to $20 \%$ and $65 \%$ of all dementia cases, respectively [1]. DLB is characterized clinically by symptoms such as visual hallucinations, Parkinsonism and fluctuating cognition in addition to cognitive impairment with typically more visuospatial and executive impairment relative to memory impairment [2]. There is some evidence that DLB patients have more rapidly progressing dementia compared to $\mathrm{AD}[3]$, and more recent studies also reported a more

\footnotetext{
* Correspondence: monica.breitve@helse-fonna.no

'Section of Mental Health Research, Clinic of Psychiatry, Helse-Fonna HF Haugesund Hospital, Post-box 2170, 5504 Haugesund, Norway

${ }^{2}$ Old Age Department, Clinic of Psychiatry, Helse-Fonna HF Haugesund

Hospital, Post-box 2170, 5504 Haugesund, Norway

Full list of author information is available at the end of the article
}

severe course with shorter survival [4], higher rate of nursing home admissions [5] and higher costs in DLB as compared to $\mathrm{AD}[6]$.

An overlap in neuropathology between AD and DLB has been noted [7]. Parkinson's disease (PD) and DLB also share some clinical and pathological features [8]. Subgroups with different cognitive profiles have been described in patients with PD [9], and there is evidence that this differentiation is related to the rate of cognitive decline [10]. Similar neuropsychologically defined subgroups may exist also in DLB [8], which could also predict differences in the rate of progression to end-stage dementia. Data supports accelerated disease progression when AD and DLB pathologies are present together [11].

To our knowledge, no systematic review has compared rate of cognitive decline in DLB versus $\mathrm{AD}$. We therefore systematically reviewed the literature to find studies assessing overall cognitive decline in DLB and AD. We 
specifically noted studies that had investigated the potential differences in cognitive decline in subgroups with DLB and the effect of employing different diagnostic criteria.

\section{Methods}

PsycINFO and Medline were searched in February 2013, using key words listed in Table 1. References from reviewed articles were also searched for relevant studies. The following inclusion criteria were used: a) paper published in a peer-reviewed journal; b) written in English; c) DLB or mixed AD/DLB compared with AD; d) application of at least one neuropsychological test, and e) at least 6 months follow up. The following exclusion criteria were used: a) drug trials, and b) survival studies with death as the only outcome.

\section{Quality assessment}

Two independent raters rated all studies with a selfdesigned quality scale and arrived at the same result. The domains, a) number of patients included; b) follow-up time; c) clinical criteria; d) autopsy, and e) neuropsychological tests) were rated on a four-point scale adapted from Aarsland et al. (2005) [12]: 0 (none), 1 (poor), 2 (fair) and 3 (good). See Table 2. Studies could be assigned 1 to 15 points.

\section{Statistical analysis}

For studies reporting mini mental state examination (MMSE) results, standardized mean difference in annual progression between DLB and AD was calculated as the difference between annual progression between the DLB and AD groups divided by the pooled standard deviation across groups in each included study. The standardized mean differences were combined in a random-effects model to obtain summary estimates of the effect in each study. The overall results from each trial were then combined using a random-effects model to obtain a pooled summary estimate of effect across all trials [13]. To assess heterogeneity, the $I^{2}$ as proposed by Higgins and colleagues [14] was chosen, indicating the percentage of total variation across studies due to heterogeneity.

\section{Results}

Of the 18 studies included in this review (see Table 3), six (36\%) reported a statistically significant difference in cognitive decline over time between AD and DLB (see Table 4). Three studies reported a faster cognitive decline on cognitive screening tests in the neuropathologically mixed AD/DLB group $[3,15,16]$ compared to those with pure $\mathrm{AD}$ or $\mathrm{DLB}$. One study reported a faster decline in DLB than in AD on verbal fluency [17], and two in AD compared to DLB on memory $[18,19]$. For a full description of neuropsychological tests used in included studies, see Table 3.

Six studies either reported annual decline in MMSE scores, or included data enabling calculation of annual decline based on reported scores. In $\mathrm{AD}$, mean annual decline was 3.3 (SD 1.7, range 1.8 to 4.9 ), and in DLB 3.4 (SD 1.4, range 1.8 to 5.8). One study also reported annual decline of 5.0 in $\mathrm{AD} / \mathrm{DLB}$ (see Figure 1). The random-effects meta-analysis revealed an overall effectsize of -0.035 (negative sign indicates faster progression in DLB) $(P=0.764 ; 95 \% \mathrm{CI}=0.261,0.192) . I^{2}$ was 50.3 , which is considered to represent moderate heterogeneity [14].

\section{Cognitive domains}

Six studies measured memory, and two reported differences in memory over time, both a faster decline in AD. Delayed recall was found to have a faster decline in $\mathrm{AD}$ compared to AD/DLB when measured with the Consortium to Establish a Registry for Alzheimer's Disease (CERAD) evaluation, with $15 \%$ of patients with $A D$ versus $32 \%$ of patients with AD/DLB remembering any item at the last evaluation [17]. Recognition was found to have a faster decline in AD compared to DLB as measured with Hopkins verbal learning test- revised (HVLT-R) (scores not available) [19]. Eight studies measuring language and ten studies measuring visuospatial ability reported no differences in rate of decline. Seven studies measured explicit executive functions, and one reported differences over time. In that study, verbal fluency was found to have a more rapid decline in DLB compared to $\mathrm{AD}$, measured with the Cambride cognitive examination (CAMCOG) (subscores not available) [17].

\section{Table 1 Search history}

\begin{tabular}{|c|c|c|}
\hline & $\begin{array}{l}\text { Medline } \\
\text { (1946 to February 2013) }\end{array}$ & $\begin{array}{l}\text { PsycINFO } \\
\text { (1806 to February 2013) }\end{array}$ \\
\hline Key words & Alzheimer's disease and Lewy body disease, or Lewy bodies & Alzheimer's disease and dementia with Lewy bodies \\
\hline Key words & $\begin{array}{l}\text { Neuropsychology, or neuropsychological tests, or Cognition, } \\
\text { or cognition disorders }\end{array}$ & $\begin{array}{l}\text { Neuropsychology, or neuropsychological assessment, } \\
\text { or neuropsychological assessment, or Cognition, or } \\
\text { cognitive impairment, or }\end{array}$ \\
\hline Key words & Disease progression, or longitudinal studies & Disease course, or disease prognosis, or longitudinal studies \\
\hline Search results & 70 & 97 \\
\hline Included & & \\
\hline
\end{tabular}


Table 2 Quality assessment criteria

\begin{tabular}{lllll}
\hline & Score & & & \\
\cline { 2 - 5 } & $\mathbf{3}$ & $\mathbf{2}$ & $\mathbf{1}$ & $\mathbf{0}$ \\
\hline Patients at baseline, number & $>151$ & 101 to 150 100 & $<50$ \\
Follow-up time, years & $>3$ or mean $\geq 3$ & 3 & 2 & $\leq 1$ \\
Clinical criteria & $\begin{array}{l}\text { Established criteria for AD + DLB } \\
\text { criteria from 2005 }\end{array}$ & $\begin{array}{l}\text { Established criteria for AD + DLB } \\
\text { criteria from 1992 or 1996 }\end{array}$ & $\begin{array}{l}\text { Used criteria for one } \\
\text { type of dementia }\end{array}$ & No criteria used \\
Autopsy, \% of participants & 100 & $>50$ & $>25$ & None \\
\hline
\end{tabular}

AD, Alzheimer's disease; DLB, dementia with Lewy bodies; BNT, Boston naming test; CERAD, Consortium to Establish a Registry for Alzheimer's Disease evaluation; DRS, dementia rating scale; ESD, extended scale for dementia; HVLT-R Hopkins verbal learning test-revised; mMMS, modified mini-mental state examination; MMSE, mini mental state examination; MTS, 37 item mental test score.

\section{Subgroups}

Two studies $[28,30]$ divided patients into two groups according to high or low visuospatial functioning. In the first study, DLB patients with a low baseline score $(<20)$ on the Wechsler intelligence scale for children-revised, block design (WISC-R) and impaired clock drawing test (CDT) had a faster decline on the dementia rating scale (DRS), compared to DLB patients with a high baseline score. In the latter study, DLB patients with a low baseline score on the Newcastle visual perception battery (NEVIP) had a faster decline in activities of daily living (ADL) than those with higher score, but no difference on any of the cognitive tests. There were no differences in the $\mathrm{AD}$ groups.

\section{Quality assessment}

The mean quality score for all the included studies was 9.4 points (SD 2.5, range 5 to 14 ) (see Table 5). Only two studies were rated fair or good on all quality measures $[26,27]$. Three studies were rated poor on one variable, but fair and good on the others $[15,16,22]$. Mean quality scores for studies that found any differences in cognitive decline was 9.8 points (SD 2.4, range 5 to 11) compared to 9.3 points (SD 2.6, range 5 to 14 ) in the group with no differences $(P=0.335)$.

\section{Clinical and neuropathological diagnostic criteria}

There were no systematical differences in clinical or neuropathological criteria between studies that found differences in cognitive decline and those who did not (see Table 6). Of 18 included studies, 16 (89\%) used National Institute of Neurological and Communication Disorders and Stroke/Alzheimer's Disease and Related Disorders Association (NINCDS/ADRDA) or CERAD clinical criteria for AD and 12 (67\%) used DLB consensus criteria, only one of them used the revised criteria from 2005. To diagnose AD neuropathologically, mainly CERAD neuropathological criteria for the diagnosis of $\mathrm{AD}$ and neuropathological DLB consensus criteria from 1996 were used. A diagnosis of mixed AD/DLB was made, if in addition to the Alzheimer's pathology the characteristic Lewy bodies were found in subcortical and cortical areas. Eleven studies (61\%) used autopsy-confirmed diagnosis on all patients. In three studies (17\%), some of the diagnoses were autopsy-confirmed. In four studies (22\%) autopsy was not performed. One of the studies used ${ }^{123}$ I-FP-CIT-SPECT only as a method of verifying of clinical diagnosis [31].

\section{Discussion}

In the 18 studies included in this review, no consistent faster rate of decline in DLB as compared to AD on cognitive screening tests was found. When combining studies that used MMSE, the most frequently used scale, a meta-analysis revealed no difference in the annual rate of cognitive decline. There were mixed findings on decline in specific cognitive domains. Two of six studies of memory found a more rapid decline in AD. Only one of seven studies of executive function found a more rapid decline in DLB, and differences in visuospatial or language tests were not found. The hypothesis of a more rapid cognitive decline in autopsied patients with both AD and DLB pathology was supported in three studies. However, findings were inconsistent and other studies did not find differences.

Differences in methods such as selection criteria, design, neuropsychological tests, dementia severity, diagnostic procedures and criteria can explain the diverse findings and lack of firm conclusions. However, quality assessment did not reveal any systematic differences between studies with high or low quality scores. There were large differences in sample sizes $(n=28$ to 315), and the studies that could not be included in the metaanalysis or used other tests than MMSE, thus, may have had varying statistical power to detect significant differences between groups. To be able to compare the overall results and draw some general conclusions it would have been ideal that uniform diagnostic criteria had been used in all the studies. Some of the studies initially included patients with a clinical diagnosis of AD only, where analyses were based on autopsy diagnosis which included both AD and DLB.

A common weakness in the included studies was the choice of neuropsychological measures. When studying 
Table 3 Study characteristics and main findings of included studies

\begin{tabular}{|c|c|c|c|c|c|}
\hline Study & $\begin{array}{l}\text { Sample, male/ } \\
\text { female ratio }(\mathrm{m} / \mathrm{f}) \text {, } \\
\text { mean age }(\mathrm{SD})\end{array}$ & $\begin{array}{l}\text { Follow-up } \\
\text { period }\end{array}$ & Neuropsychological tests & $\begin{array}{l}A D \text { versus } D L B \\
\text { comparison }\end{array}$ & Test scores, mean (SD) \\
\hline \multirow{6}{*}{$\begin{array}{l}\text { McKeith et al., } \\
1992 \text { [20] }\end{array}$} & AD 37 & \multirow{6}{*}{$\begin{array}{l}\text { Baseline and } \\
\text { late stage }\end{array}$} & \multirow[t]{6}{*}{ MTS } & \multirow[t]{6}{*}{ No significant difference } & MTS baseline \\
\hline & $m / f 13 / 24$ & & & & AD 15.9 (1.8) \\
\hline & y $74.7(0.9)$ & & & & SDLT 24.5 (1.7) \\
\hline & SDLT 21 & & & & MTS late stage \\
\hline & $\mathrm{m} / \mathrm{f} 12 / 9$ & & & & AD $9.3(2.1)$ \\
\hline & y $73.3(1.6)$ & & & & SDLT 18.2 (2.3) \\
\hline \multirow[t]{10}{*}{$\begin{array}{l}\text { Ballard et al., } \\
1996 \text { [17] }\end{array}$} & AD 53 & \multirow[t]{10}{*}{$1 y$} & \multirow[t]{10}{*}{ CAMCOG } & \multirow[t]{10}{*}{$\begin{array}{l}\text { SDLT faster decline of } \\
\text { verbal fluency }\end{array}$} & Scores for subtests n/a \\
\hline & $\mathrm{m} / \mathrm{f}, \mathrm{n} / \mathrm{a}$ & & & & \\
\hline & $\mathrm{Y}, \mathrm{n} / \mathrm{a}$ & & & & CAMCOG total, baseline \\
\hline & \multirow[t]{2}{*}{ SDLT 7} & & & & AD $42.7(17.9)$ \\
\hline & & & & & SDLT 47.7 (18.0) \\
\hline & $\mathrm{m} / \mathrm{f}, \mathrm{n} / \mathrm{a}$ & & & & CAMCOG mean annual decline \\
\hline & $Y, n / a$ & & & & \\
\hline & $\operatorname{VaD} 14$ & & & & AD 13.2 (12.6) \\
\hline & $\mathrm{m} / \mathrm{f}, \mathrm{n} / \mathrm{a}$ & & & & SDLT 27.0 (19.8) \\
\hline & $\mathrm{Y}, \mathrm{n} / \mathrm{a}$ & & & & \\
\hline \multirow{7}{*}{$\begin{array}{l}\text { Ballard et al., } \\
1998[21]\end{array}$} & AD 30 & \multirow[t]{7}{*}{$1 y$} & \multirow[t]{7}{*}{ MMSE } & \multirow[t]{7}{*}{ No significant difference } & MMSE baseline \\
\hline & \multirow[t]{2}{*}{ m/f 9/21 } & & & & AD 13.9 \\
\hline & & & & & DLB 14.9 \\
\hline & y 81.7 & & & & MMSE mean annual decline \\
\hline & DLB 42 & & & & AD 4.1 \\
\hline & $m / f 19 / 24$ & & & & DLB 3.9 \\
\hline & y 73.6 & & & & \\
\hline \multirow{19}{*}{$\begin{array}{l}\text { Olichney et } \\
\text { al., } 1998 \text { [3] }\end{array}$} & AD 148 & \multirow[t]{19}{*}{ Mean 3 y } & \multirow[t]{19}{*}{ MMSE } & \multirow[t]{19}{*}{ LBV faster decline } & MMSE baseline \\
\hline & $\mathrm{m} / \mathrm{f} 80 / 68$ & & & & \\
\hline & y $74.0(7.9)$ & & & & AD $17.8(6.0)$ \\
\hline & LBV 40 & & & & LBV 18.2 (5.5) \\
\hline & $m / f$ 25/15 & & & & MMSE 1 y $(n=136 / 35)$ \\
\hline & \multirow[t]{14}{*}{ y $72.4(6.5)$} & & & & AD $14.3(7.2)$ \\
\hline & & & & & LBV 12.5 (7.5) \\
\hline & & & & & MMSE 2 y $(n=93 / 17)$ \\
\hline & & & & & AD $12.3(7.9)$ \\
\hline & & & & & LBV 8.1 (6.3) \\
\hline & & & & & MMSE 3 y $(n=59 / 12)$ \\
\hline & & & & & AD $10.1(8.4)$ \\
\hline & & & & & LBV 4.5 (6.5) \\
\hline & & & & & MMSE 4 y $(n=35 / 4)$ \\
\hline & & & & & AD 9.1 (7.9) \\
\hline & & & & & LBV 2.5 (3.0) \\
\hline & & & & & MMSE mean annual decline \\
\hline & & & & & AD 4.1 (3.0) \\
\hline & & & & & LBV 5.8 (4.5) \\
\hline
\end{tabular}


Table 3 Study characteristics and main findings of included studies (Continued)

\begin{tabular}{|c|c|c|c|c|c|}
\hline \multirow{6}{*}{$\begin{array}{l}\text { Heyman et al., } \\
1999 \text { [18] }\end{array}$} & AD 74 & \multirow{6}{*}{$\begin{array}{l}\text { Annual } \\
\text { controls }\end{array}$} & \multirow{6}{*}{$\begin{array}{l}\text { CERAD (including CDT, } \\
\text { calculation test, serial } \\
\text { subtraction, CDR, BNT, MMSE, } \\
10 \text {-item word list memory, } \\
\text { recall and recognition, } \\
\text { constructional praxis, two of } \\
\text { the six items of the } \\
\text { orientation-memory- } \\
\text { concentration test) }\end{array}$} & \multirow{6}{*}{$\begin{array}{l}\text { AD faster decline in } \\
\text { delayed recall }\end{array}$} & \multirow{6}{*}{$\begin{array}{l}32 \% \text { of } L B V \text { versus } 15 \% \text { of } A D \\
\text { remembered any item on } \\
\text { word list recall at last } \\
\text { evaluation }\end{array}$} \\
\hline & $m / f 47 / 27$ & & & & \\
\hline & y $41 \%>74$ y & & & & \\
\hline & AD/LBV 27 & & & & \\
\hline & $m / f 14 / 13$ & & & & \\
\hline & y $37 \%>74$ y & & & & \\
\hline \multirow{6}{*}{$\begin{array}{l}\text { Lopez et al., } \\
2000 \text { [22] }\end{array}$} & AD 98 & \multirow{6}{*}{$\begin{array}{l}\text { Mean } \\
59 \text { months }\end{array}$} & \multirow[t]{6}{*}{ MMSE } & \multirow[t]{6}{*}{ No significant difference } & \multirow[t]{2}{*}{ MMSE baseline } \\
\hline & $\mathrm{m} / \mathrm{f} 50 / 48$ & & & & \\
\hline & y $70.8(9.4)$ & & & & AD $16.0(6.5)$ \\
\hline & AD/DLB 44 & & & & AD/DLB $16.2(5.1)$ \\
\hline & $m / f$ 20/24 & & & & \\
\hline & y $72.3(6.0)$ & & & & \\
\hline \multirow{6}{*}{$\begin{array}{l}\text { Stern et al., } \\
2001 \text { [23] }\end{array}$} & AD 32 & \multirow{6}{*}{$\begin{array}{l}\text { Annual } \\
\text { controls, } \\
\text { longest } 9.9 \text { y }\end{array}$} & \multirow{6}{*}{$\begin{array}{l}\text { mMMSE (including WAIS-R } \\
\text { digit span forward, backward, } \\
\text { attention, calculation, general } \\
\text { knowledge, language, } \\
\text { construction), CDR }\end{array}$} & \multirow[t]{6}{*}{ No significant difference } & mMMSE baseline \\
\hline & $m / f 16 / 16$ & & & & AD 36.7 (6.3) \\
\hline & y $73.0(9.0)$ & & & & LBV 37.3 (6.2) \\
\hline & LBV 19 & & & & mMMSE mean annual decline \\
\hline & $\mathrm{m} / \mathrm{f} 17 / 2$ & & & & 3.6 (both groups) \\
\hline & у $73.6(6.8)$ & & & & \\
\hline \multirow{16}{*}{$\begin{array}{l}\text { Ballard et al., } \\
2001 \text { [24] }\end{array}$} & AD 101 & \multirow[t]{16}{*}{$1 y$} & \multirow[t]{16}{*}{ MMSE, CAMCOG } & \multirow[t]{16}{*}{ No significant difference } & MMSE $n=203$ \\
\hline & $\mathrm{m} / \mathrm{f} 30 / 71$ & & & & MMSE baseline \\
\hline & probable AD & & & & prob AD 17.7 (5.1) \\
\hline & $61 \mathrm{~m} / \uparrow 1 / / 44$ & & & & poss AD $17.2(5.2)$ \\
\hline & y $81.9(4.8)$ & & & & DLB $15.6(7.0)$ \\
\hline & possible AD 40 & & & & MMSE mean annual decline \\
\hline & $m / f 13 / 27$ & & & & \\
\hline & у $79.0(7.8)$ & & & & AD 4.9 (3.6) \\
\hline & DLB 64 & & & & DLB $4.3(4.2)$ \\
\hline & $m / f 26 / 38$ & & & & CAMCOG $n=154$ \\
\hline & y $76.6(7.7)$ & & & & Baseline 57.5 (18.8) \\
\hline & $\operatorname{VaD} 38$ & & & & CAMCOG mean annual decline \\
\hline & $\mathrm{m} / \mathrm{f} 22 / 16$ & & & & \\
\hline & y $76.8(7.7)$ & & & & Probable AD 15.0 (10.1) \\
\hline & & & & & Possible AD 14.4 (9.8) \\
\hline & & & & & DLB 11.9 (12.2) \\
\hline \multirow{9}{*}{$\begin{array}{l}\text { Helmes et al., } \\
2003 \text { [25] }\end{array}$} & AD 15 & \multirow[t]{9}{*}{50 months } & \multirow[t]{9}{*}{ ESD } & \multirow[t]{9}{*}{ No significant difference } & \multirow[t]{9}{*}{ Scores n/a } \\
\hline & $\mathrm{m} / \mathrm{f} 9 / 6$ & & & & \\
\hline & у $70.3(7.6)$ & & & & \\
\hline & AD/DLB 8 & & & & \\
\hline & $m / f 5 / 3$ & & & & \\
\hline & y $69.3(11.2)$ & & & & \\
\hline & DLB 7 & & & & \\
\hline & $m / f 5 / 2$ & & & & \\
\hline & y 69.1 (4.1) & & & & \\
\hline
\end{tabular}


Table 3 Study characteristics and main findings of included studies (Continued)

\begin{tabular}{|c|c|c|c|c|c|}
\hline \multirow[t]{8}{*}{$\begin{array}{l}\text { Johnson et al., } \\
2005 \text { [26] }\end{array}$} & AD 66 & $\begin{array}{l}\text { Annual } \\
\text { controls, }\end{array}$ & \multirow{8}{*}{$\begin{array}{l}\text { WMS (digits forward, } \\
\text { backward, logical memory and } \\
\text { associate learning), BVRT, word } \\
\text { fluency, BNT, WAIS (Digit } \\
\text { Symbol and Block Design), } \\
\text { TMT A, Crossing Off, CDR }\end{array}$} & \multirow[t]{8}{*}{ No significant difference } & \multirow{8}{*}{$\begin{array}{l}\text { Follow-up scores } n / a \text {. For } \\
\text { baseline scores for all tests see } \\
\text { article }\end{array}$} \\
\hline & m/f 39/27 & \multirow{7}{*}{$\begin{array}{l}1 \text { to } 20 \\
\text { assessments }\end{array}$} & & & \\
\hline & y $77.0(8.1)$ & & & & \\
\hline & AD/DLB 57 & & & & \\
\hline & $\mathrm{m} / \mathrm{f} 31 / 26$ & & & & \\
\hline & y $75.2(9.7)$ & & & & \\
\hline & DLB 9 & & & & \\
\hline & $\begin{array}{l}\mathrm{m} / \mathrm{f} 8 / 1 \text {, age } 72.6 \\
(5.7)\end{array}$ & & & & \\
\hline \multirow[t]{16}{*}{$\begin{array}{l}\text { Kraybill et al., } \\
2005 \text { [15] }\end{array}$} & AD 48 & \multirow[t]{16}{*}{$\begin{array}{l}\text { Annual } \\
\text { controls }\end{array}$} & \multirow[t]{16}{*}{ MMSE, DRS } & \multirow[t]{16}{*}{$\begin{array}{l}\mathrm{AD} / \mathrm{LBP} \text { faster decline than } \\
\mathrm{AD} \text { and } \mathrm{LBP}\end{array}$} & MMSE baseline \\
\hline & $\mathrm{m} / \mathrm{f} 18 / 30$ & & & & AD 20.6 (3.9) \\
\hline & $y$ at onset 77.5 & & & & AD/LBP 20.7 (3.7) \\
\hline & $(7.34)$ & & & & LBP 20.7 (3.8) \\
\hline & AD/LBP 65 & & & & MMSE mean annual decline \\
\hline & $m / f$ 24/41 & & & & AD $3.5(0.4)$ \\
\hline & \multirow{2}{*}{$\begin{array}{l}y \text { at onset } 74.8 \\
(6.6)\end{array}$} & & & & AD/LBP $5.0(0.5)$ \\
\hline & & & & & LBP $3.4(0.7)$ \\
\hline & LBP 22 & & & & DRS baseline \\
\hline & $\mathrm{m} / \mathrm{f} 16 / 6$ & & & & AD 114.7 (2.1) \\
\hline & \multirow{6}{*}{$\begin{array}{l}y \text { at onset } 76.5 \\
(5.3)\end{array}$} & & & & AD/LBP 114.2 (1.8) \\
\hline & & & & & LBP $114.2(2.7)$ \\
\hline & & & & & DRS mean annual decline \\
\hline & & & & & AD $9.6(1.5)$ \\
\hline & & & & & AD/LBP 15.3 (1.9) \\
\hline & & & & & LBP $8.8(1.7)$ \\
\hline \multirow{7}{*}{$\begin{array}{l}\text { Stavitsky et al., } \\
2006 \text { [19] }\end{array}$} & AD 55 & \multirow[t]{7}{*}{ Mean $3 y$} & \multirow{7}{*}{$\begin{array}{l}\text { mMMSE (incl WAIS-R digit Span } \\
\text { forward, backward, attention, } \\
\text { calculation, general knowledge, } \\
\text { language, construction), HVLT-R }\end{array}$} & \multirow{7}{*}{$\begin{array}{l}\text { AD faster decline on } \\
\text { recognition. }\end{array}$} & mMMSE baseline \\
\hline & \multirow[t]{2}{*}{$m / f 21 / 34$} & & & & AD $39.0(7.6)$ \\
\hline & & & & & DLB 38.1 (8.3) \\
\hline & y $73.1(8.3)$ & & & & HVLT-R n/a \\
\hline & DLB 28 & & & & \\
\hline & $m / f 19 / 9$ & & & & \\
\hline & y $73.5(7.6)$ & & & & \\
\hline \multirow{7}{*}{$\begin{array}{l}\text { Williams et al., } \\
2006 \text { [27] }\end{array}$} & AD 252 & \multirow[t]{6}{*}{$<5 y$} & \multirow{7}{*}{$\begin{array}{l}\text { MMSE, CDR, WMS (mental } \\
\text { control, logical memory, digit } \\
\text { span forward and backward, } \\
\text { associate learning), BVRT, WAIS } \\
\text { (information, digit symbol, } \\
\text { block design), word fluency, } \\
\text { BNT, Crossing off, TMT A }\end{array}$} & \multirow[t]{7}{*}{ No significant difference. } & \multirow[t]{6}{*}{ Scores n/a } \\
\hline & $\mathrm{m} / \mathrm{f} 95 / 157$ & & & & \\
\hline & y $77.8(9.5)$ & & & & \\
\hline & DLB 63 & & & & \\
\hline & $\mathrm{m} / \mathrm{f} 38 / 25$ & & & & \\
\hline & y $73.5(8.7)$ & & & & \\
\hline & $\mathrm{AD} 44$ & $2 y$ & & & DRS baseline \\
\hline
\end{tabular}


Table 3 Study characteristics and main findings of included studies (Continued)

\begin{tabular}{|c|c|c|c|c|c|}
\hline \multirow{10}{*}{$\begin{array}{l}\text { Hamilton et } \\
\text { al., } 2008 \text { [28] }\end{array}$} & $m / f$ 20/24 & & \multirow{10}{*}{$\begin{array}{l}\text { DRS, WISC-R (block design), } \\
\text { CDT copy, BNT }\end{array}$} & \multirow{10}{*}{$\begin{array}{l}\text { Poor baseline visuospatial } \\
\text { skills (block design }<20 \text {, } \\
\text { CDT copy }<3 \text { ) were } \\
\text { strongly associated with } \\
\text { faster decline in DLB, but } \\
\text { not AD. }\end{array}$} & AD 114.4 (15.4) \\
\hline & y $72.0(5.6)$ & & & & DLB 109.5 (11.4) \\
\hline & DLB 22 & & & & DRS 1 y mean decline \\
\hline & $\mathrm{m} / \mathrm{f} 14 / 8$ & & & & \\
\hline & y $73.4(6.2)$ & & & & AD 7.9 (11.6) \\
\hline & & & & & DLB 17 (24.2) \\
\hline & & & & & DRS 2 y mean decline \\
\hline & & & & & AD $23.9(24.7)$ \\
\hline & & & & & DLB 39.3 (35.1) \\
\hline & & & & & Other scores n/a \\
\hline \multirow{19}{*}{$\begin{array}{l}\text { Hanyu et al., } \\
2009 \text { [29] }\end{array}$} & AD 111 & \multirow[t]{19}{*}{$5 y$} & \multirow[t]{19}{*}{ MMSE } & \multirow[t]{19}{*}{ No significant difference } & MMSE \\
\hline & $\mathrm{m} / \mathrm{f} 37 / 74$ & & & & Baseline $n=111 / 56$ \\
\hline & y $77.5(6.2)$ & & & & AD $20.3(3.7)$ \\
\hline & DLB 56 & & & & DLB 20.7 (3.8) \\
\hline & $\mathrm{m} / \mathrm{f} 30 / 26$ & & & & 1 y $n=111 / 56$ \\
\hline & y $78.1(5.2)$ & & & & AD $19.4(4.8)$ \\
\hline & & & & & DLB 20.5 (4.2) \\
\hline & & & & & 2 y $n=102 / 40$ \\
\hline & & & & & $\mathrm{AD} 17.7(5.2)$ \\
\hline & & & & & DLB $18.0(4.8)$ \\
\hline & & & & & 3 y $n=72 / 25$ \\
\hline & & & & & AD $16.2(5.0)$ \\
\hline & & & & & DLB $17.0(5.3)$ \\
\hline & & & & & 4 y $n=51 / 19$ \\
\hline & & & & & AD $14.2(4.5)$ \\
\hline & & & & & DLB $13.4(4.0)$ \\
\hline & & & & & 5 y $n=16 / 5$ \\
\hline & & & & & AD $11.4(5.2)$ \\
\hline & & & & & DLB $10.6(4.0)$ \\
\hline \multirow{9}{*}{$\begin{array}{l}\text { Nelson et al., } \\
2009 \text { [16] }\end{array}$} & AD 107 & \multirow[t]{9}{*}{ Mean 4 y } & \multirow[t]{9}{*}{ MMSE } & \multirow{9}{*}{$\begin{array}{l}\text { AD/DLB had a faster } \\
\text { decline than DLB and AD. }\end{array}$} & MMSE baseline $\mathrm{n} / \mathrm{a}$ \\
\hline & $m / f n / a$ & & & & MMSE final \\
\hline & y n/a & & & & AD 10.7 (8.6) \\
\hline & AD/DLB 27 & & & & AD/DLB 10.6 (8.6) \\
\hline & $m / f n / a$ & & & & DLB $15.6(8.7)$ \\
\hline & y n/a & & & & \\
\hline & DLB 9 & & & & \\
\hline & $m / f n / a$ & & & & \\
\hline & y $n / a$ & & & & \\
\hline
\end{tabular}


Table 3 Study characteristics and main findings of included studies (Continued)

\begin{tabular}{|c|c|c|c|c|c|}
\hline \multirow{13}{*}{$\begin{array}{l}\text { Wood et al., } \\
2012 \text { [30] }\end{array}$} & $\mathrm{AD} 16$ & $1 y$ & MMSE, CAMCOG, NEVIP & No significant difference. & MMSE baseline \\
\hline & $\mathrm{m} / \mathrm{f} 12 / 4$ & & & & AD $21.3(3.2)$ \\
\hline & y $78.9(6.1)$ & & & & DLB 24.5 (3.3) \\
\hline & DLB 10 & & & & MMSE decline from baseline \\
\hline & $\mathrm{m} / \mathrm{f} 9 / 1$ & & & & \\
\hline & у 78.2 (7.4). & & & & AD 2.1 (3.6) \\
\hline & Controls 28 & & & & DLB $1.8(3.1)$ \\
\hline & $m / f \quad 16 / 12$ & & & & CAMCOG baseline \\
\hline & y 79.5 & & & & AD $71.4(9.7)$ \\
\hline & & & & & DLB 79.1 (12.0) \\
\hline & & & & & $\begin{array}{l}\text { CAMCOG decline from } \\
\text { baseline }\end{array}$ \\
\hline & & & & & AD $7.4(10.7)$ \\
\hline & & & & & DLB $4.3(7.3)$ \\
\hline \multirow{13}{*}{$\begin{array}{l}\text { Walker et al., } \\
2012 \text { [31] }\end{array}$} & AD 100 & $1 y$ & MMSE, CAMCOG-R, VOSP, CDR & No significant difference. & MMSE baseline \\
\hline & $m / f 48 / 52$ & & & & AD 21.5 (4.5) \\
\hline & y 74,9 & & & & DLB 21.4 (3.9) \\
\hline & DLB 58 & & & & MMSE follow up $(n=81 / 33)$ \\
\hline & $m / f 37 / 21$ & & & & AD $19.0(6.2)$ \\
\hline & y 74,2 & & & & DLB 18.5 (6.0) \\
\hline & & & & & CAMCOG-R baseline \\
\hline & & & & & AD 66.3 (15.6) \\
\hline & & & & & DLB 66.0 (13.5) \\
\hline & & & & & CAMCOG-R follow up \\
\hline & & & & & $(n=81 / 33)$ \\
\hline & & & & & AD $59.6(20.3)$ \\
\hline & & & & & DLB 56.3 (19.7) \\
\hline
\end{tabular}

AD, Alzheimer's disease; DLB, dementia with Lewy bodies; LBP, Lewy body pathology; LBV, Lewy body variant; $n / a$, not available; SDLT, senile dementia of Lewy body type; VaD, vascular dementia; $y$, years; BNT, Boston naming test; BVRT, Benton visual retention test; CAMCOG, Cambridge cognitive examination; CAMCOG-R, Cambridge cognitive examination-revised; CDR, clinical dementia rating; CDT, clock drawing test; CERAD, Consortium to Establish a Registry for Alzheimer's Disease evaluation; DRS, dementia rating scale; ESD, extended scale for dementia; HVLT-R, Hopkins verbal learning test-revised; MMSE, mini mental state examination; mMMS, modified mini-mental state examination; MTS, 37-item mental test score; NEVIP, Newcastle visual perception battery; TMT A, trail making test A; VOSP, visual object and space perception battery; WAIS, Wechsler adult intelligence scale; WISC-R, Wechsler intelligence scale for children-revised; WMS, Wechsler memory scale.

Table 4 Studies reporting differences in cognitive decline

\begin{tabular}{llll}
\hline Study & Cognitive function & Impairment & Contrast group \\
\hline Olichney et al., 1998 [3] & Total score & AD/DLB & AD \\
Kraybill et al I., 2005 [15] & Total score & AD/DLB & AD and DLB \\
Nelson et al., 2009 [16] & Total score & AD/DLB & AD and DLB \\
Heyman et al., 1999 [18] & Delayed recall & AD & AD/DLB \\
Stavitsky et al., 2006 [19] & Recognition & AD & DLB \\
Ballard et al., 1996 [17] & Verbal fluency & DLB & AD \\
\hline
\end{tabular}

AD, Alzheimer's disease; AD/DLB, mixed pathology; DLB, dementia with Lewy bodies;

CAMCOG, Cambridge cognitive examination; CERAD, Consortium to Establish a Registry for Alzheimer's Disease evaluation; DRS, dementia rating scale; HVLT-R Hopkins verbal learning test-revised; MMSE, mini mental state examination. 


\begin{tabular}{|c|c|c|c|c|c|c|c|}
\hline \multirow[t]{2}{*}{ Study name } & \multicolumn{7}{|c|}{ Statistics for each study } \\
\hline & $\begin{array}{l}\text { Std diff } \\
\text { in means }\end{array}$ & $\begin{array}{l}\text { Standard } \\
\text { error }\end{array}$ & Variance & $\begin{array}{l}\text { Lower } \\
\text { limit }\end{array}$ & $\begin{array}{l}\text { Upper } \\
\text { limit }\end{array}$ & Z-Value & p-Value \\
\hline Kraybill & 0,034 & 0,257 & 0,066 & $-0,471$ & 0,539 & 0,132 & 0,89 \\
\hline Ballard 2001 & 0,157 & 0,160 & 0,026 & $-0,157$ & 0,470 & 0,979 & 0,328 \\
\hline Hanyu & 0,191 & 0,164 & 0,027 & $-0,131$ & 0,512 & 1,162 & 0,245 \\
\hline Wood & 0,088 & 0,403 & 0,163 & $-0,702$ & 0,878 & 0,218 & 0,827 \\
\hline Walker & $-0,122$ & 0,165 & 0,027 & $-0,445$ & 0,202 & $-0,736$ & 0,46 \\
\hline \multirow[t]{2}{*}{ Olichney } & $-0,487$ & 0,180 & 0,032 & $-0,839$ & $-0,134$ & $-2,705$ & 0,00 \\
\hline & $-0,035$ & 0,116 & 0,013 & $-0,261$ & 0,192 & $-0,300$ & \\
\hline
\end{tabular}

Std diff in means and $95 \% \mathrm{Cl}$

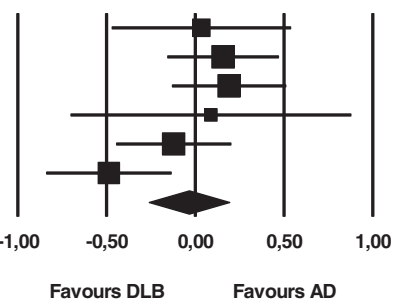

Figure 1 Forrest plot of annual progression of mini-mental state examination scores. The random-effects meta-analysis revealed an overall effect-size of -0.035 (negative sign indicates faster progression in dementia with Lewy bodies (DLB) $(P=0.764 ; 95 \% \mathrm{Cl}=0.261,0.192)$. AD,

Alzheimer's disease.

cognitive decline over time, cognitive tests that are designed for a specific cognitive domain are required. Screening tests or batteries that use a total score only, often designed for purposes other than research are less suitable. In this review, the MMSE was the most used test, either alone, or in combination with others. The MMSE may not be an optimal measure, especially when using only the total score and not separate subscores for different cognitive domains, as AD and DLB have different cognitive profiles at onset [32]. This difference in cognitive profile leads to difficulties in choosing an optimal cognitive screening instrument to compare AD and DLB. The MMSE is heavily based on memory and language and is thus more sensitive to changes in AD than in DLB [33].
DLB is associated with a more severe visuospatial deficit than AD [32,34], but only 1 of 30 points on the MMSE comes from a measure of visuospatial functioning. MMSE may also be less than optimal because of the ceiling and floor effect [35], which refers to a test being too easy or too difficult to discriminate below or above a certain point, which is a common problem when testing people with dementia. In one of the reviewed studies the children's version of the Wechsler intelligence scale was used to avoid this. The test then lacks age adjusted norms, but it gains a wider range in scores, and therefore can monitor the cognitive decline over a longer period of time. Studies differed also with regard to the time period of observation, from 1 to

Table 5 Quality assessment results

\begin{tabular}{|c|c|c|c|c|c|c|}
\hline Study & Sum & Patients & Neuropsychological tests & Time & Autopsy & Clinical criteria \\
\hline Williams et al., 2006 [27] & 14 & 3 & 3 & 3 & 3 & 2 \\
\hline Johnson et al., 2005 [26] & 13 & 2 & 3 & 3 & 3 & 2 \\
\hline Heyman et al., 1999 [18] & 11 & 1 & 3 & 3 & 3 & 1 \\
\hline Lopez et al., 2000 [22] & 11 & 2 & 1 & 3 & 3 & 2 \\
\hline Kraybill et al., 2005 [15] & 11 & 2 & 2 & 3 & 3 & 1 \\
\hline Olichney et al., 1998 [3] & 11 & 3 & 1 & 3 & 3 & 1 \\
\hline Nelson et al., 2009 [16] & 11 & 2 & 1 & 3 & 3 & 2 \\
\hline Stern et al., 2001 [23] & 10 & 1 & 2 & 3 & 3 & 1 \\
\hline Stavitsky et al., 2006 [19] & 10 & 1 & 3 & 3 & 1 & 2 \\
\hline Hamilton et al., 2008 [28] & 10 & 1 & 3 & 1 & 3 & 2 \\
\hline Helmes et al., 2003 [25] & 9 & 0 & 2 & 3 & 3 & 1 \\
\hline Hanyu et al., 2009 [29] & 9 & 3 & 1 & 3 & 0 & 2 \\
\hline McKeith et al., 1992 [20] & 8 & 1 & 1 & 3 & 3 & 0 \\
\hline Ballard et al., 2001 [24] & 8 & 3 & 2 & 0 & 1 & 2 \\
\hline Walker et al., 2012 [31] & 8 & 3 & 3 & 0 & 0 & 2 \\
\hline Wood et al., 2012 [30] & 6 & 0 & 3 & 0 & 0 & 3 \\
\hline Ballard et al., 1998 [21] & 5 & 1 & 1 & 0 & 1 & 2 \\
\hline Ballard et al., 1996 [17] & 5 & 1 & 2 & 0 & 0 & 2 \\
\hline
\end{tabular}


Table 6 Clinical and neuropathological criteria

\begin{tabular}{|c|c|c|c|c|c|}
\hline Study & Sample & Database & Neuropathological criteria & Autopsy & Dementia criteria \\
\hline \multirow{3}{*}{$\begin{array}{l}\text { McKeith et al., } \\
1992[20]\end{array}$} & AD 37 & \multirow[t]{3}{*}{ Newcastle, UK } & \multirow{2}{*}{$\begin{array}{l}\text { AD: plaque/tangle quantification, } \\
\mathrm{H}+\mathrm{E}, \mathrm{CFV} \text {, Loyez, Palmgren. }\end{array}$} & \multirow[t]{3}{*}{ All } & \multirow[t]{3}{*}{ DLB: proposed consensus (1992) } \\
\hline & SDLT & & & & \\
\hline & 21 & & LB: $H+E$, pholxine, erythrosin & & \\
\hline \multirow{3}{*}{$\begin{array}{l}\text { Ballard et al., } \\
1996[17]\end{array}$} & AD 53 & \multirow{3}{*}{$\begin{array}{l}\text { West Midlands and } \\
\text { Bristol, UK }\end{array}$} & & \multirow[t]{3}{*}{0} & AD: NINCDS/ADRDA (1984) \\
\hline & SDLT 7 & & & & \multirow{2}{*}{$\begin{array}{l}\text { DLB: McKeith, operational criteria for senile } \\
\text { dementia of Lewy body type (1992) }\end{array}$} \\
\hline & VaD 14 & & & & \\
\hline \multirow{3}{*}{$\begin{array}{l}\text { Ballard et al., } \\
1998[21]\end{array}$} & AD 30 & \multirow{3}{*}{$\begin{array}{l}\text { Newcastle General } \\
\text { Hospital, UK }\end{array}$} & AD: CERAD, plaque - Braunmuhl & \multirow[t]{3}{*}{19} & AD: NINCDS/ADRDA (1984) \\
\hline & DLB 42 & & & & \multirow{2}{*}{$\begin{array}{l}\text { DLB: McKeith, operational criteria for senile } \\
\text { dementia of Lewy body type (1992) }\end{array}$} \\
\hline & & & $\begin{array}{l}\text { LB: consensus criteria (1996), } \\
\text { ubiquitin, anti-tau2, anti-Alz50, } \\
\text { anti-AT8 to detect and distinguish } \\
\text { cortical LB }\end{array}$ & & \\
\hline \multirow{3}{*}{$\begin{array}{l}\text { Olichney et al., } \\
1998 \text { [3] }\end{array}$} & AD 148 & Cohort from: & AD: CERAD, ADRC & \multirow[t]{3}{*}{ All } & AD: NINCDS/ADRDA (1984), \\
\hline & LBV 40 & $\begin{array}{l}\text { Univeristy of California, } \\
\text { San Diego Alzheimer's } \\
\text { Disease Research Center, } \\
\text { USA; }\end{array}$ & $\begin{array}{l}\text { LB: ubiquitin, } \mathrm{H}+\mathrm{E} \text { (brainstem, } \\
\text { cerebral cortex) }\end{array}$ & & DSM-III for dementia \\
\hline & & $\begin{array}{l}\text { CERAD centers, } \\
\text { multinational }\end{array}$ & & & \\
\hline \multirow{2}{*}{$\begin{array}{l}\text { Heyman et al., } \\
1999[18]\end{array}$} & AD 74 & \multirow{2}{*}{$\begin{array}{l}\text { Subjects with } \\
\text { premortem diagnosis of } \\
\text { probable and possible } \\
\text { AD from } 24 \text { centers } \\
\text { participating in CERAD, } \\
1986 \text { to } 1995 \text {, USA }\end{array}$} & AD: CERAD & \multirow[t]{2}{*}{ All } & \multirow[t]{2}{*}{ AD: NINCDS/ADRDA (1984) } \\
\hline & $\begin{array}{l}\mathrm{AD} / \mathrm{LBV} \\
27\end{array}$ & & $\begin{array}{l}\text { LB: consensus criteria (1996), } \\
\text { modified (brainstem, limbic/ } \\
\text { transitional and noecortical). }\end{array}$ & & \\
\hline \multirow{2}{*}{$\begin{array}{l}\text { Lopez et al., } \\
2000[22]\end{array}$} & AD 98 & \multirow{2}{*}{$\begin{array}{l}\text { University of Pittsburg } \\
1983 \text { to } 1998 \text {, USA }\end{array}$} & AD: CERAD, NIA-RI & \multirow[t]{2}{*}{ All } & AD: NINCDS/ADRDA (1984) \\
\hline & $\begin{array}{l}\mathrm{AD} / \mathrm{DLB} \\
44\end{array}$ & & $\begin{array}{l}\text { LB: } H+E \text {, ubiqutin ( } S N \text {, neocortex, } \\
\text { limbic areas) }\end{array}$ & & DLB: consensus criteria (1996) \\
\hline \multirow{6}{*}{$\begin{array}{l}\text { Stern et al. } \\
2001 \text { [23] }\end{array}$} & $\mathrm{AD} 32$ & \multirow{2}{*}{$\begin{array}{l}\text { From cohort of } 236 \\
\text { patients with probable } \\
A D\end{array}$} & AD: CERAD & \multirow[t]{6}{*}{ All } & \multirow[t]{6}{*}{ AD: NINCDS/ADRDA (1984) } \\
\hline & LBV 19 & & \multirow{5}{*}{$\begin{array}{l}\text { LB: semi quantitative ubiquitin (SN, } \\
\text { hippocampus, cingulate gyrus, insula } \\
\text { cortex) }\end{array}$} & & \\
\hline & & Recruited: & & & \\
\hline & & $\begin{array}{l}\text { Columbia University } \\
\text { College, New York, USA }\end{array}$ & & & \\
\hline & & $\begin{array}{l}\text { Johns Hopkins } \\
\text { University, Baltimore, } \\
\text { USA }\end{array}$ & & & \\
\hline & & $\begin{array}{l}\text { Massachusetts General } \\
\text { Hospital, Boston, USA }\end{array}$ & & & \\
\hline \multirow{3}{*}{$\begin{array}{l}\text { Ballard et al., } \\
2001[24]\end{array}$} & AD 101 & Cohort of 227 patients & \multirow{2}{*}{$\begin{array}{l}\text { AD: CERAD, plaque - Braunmuhl } \\
\text { stain, tangle - modified Palmgren }\end{array}$} & \multirow[t]{3}{*}{50} & AD: NINCDS/ADRDA (1984) \\
\hline & DLB 64 & Institute of the Health of & & & DLB: consensus criteria (1996) \\
\hline & $\operatorname{VaD} 38$ & $\begin{array}{l}\text { the Elderly (IHE), } \\
\text { Newcastle, UK }\end{array}$ & $\begin{array}{l}\text { LB: consensus criteria (1996), } \\
\text { ubiquitin, anti-tau2, anti-Alz50, } \\
\text { anti-AT8 to detect and distinguish } \\
\text { cortical LB }\end{array}$ & & \\
\hline Helmes et al., & $\mathrm{AD} 15$ & University of Western & No criteria are referred to. Only & All & Not specified. \\
\hline & $\begin{array}{l}\text { AD/DLB } \\
8\end{array}$ & $\begin{array}{l}\text { Ontario Dementia Study, } \\
\text { Canada }\end{array}$ & $\begin{array}{l}\text { referred to LB staining methods } \\
\text { (Bielschovsky, anti-ubiquitin, } \\
\text { anti-synuclein). }\end{array}$ & & \\
\hline & DLB 7 & & & & \\
\hline Johnson et al., & AD 66 & Washington University, & AD: NIA-RI quantification of diffuse & All & AD: NINCDS/ADRDA (1984) \\
\hline & $\begin{array}{l}\mathrm{AD} / \mathrm{DLB} \\
57\end{array}$ & & $\begin{array}{l}\text { and neuritic depositions in to } \\
\text { cortical regions }\end{array}$ & & $\begin{array}{l}\text { DLB: consensus criteria (1996) or McKeith, } \\
\text { operational criteria for senile dementia of } \\
\text { Lewy body type (1992) }\end{array}$ \\
\hline & DLB 9 & & LB: synuclein & & \\
\hline
\end{tabular}


Table 6 Clinical and neuropathological criteria (Continued)

\begin{tabular}{|c|c|c|c|c|c|}
\hline \multirow{3}{*}{$\begin{array}{l}\text { Kraybill et al., } \\
2005 \text { [15] }\end{array}$} & AD 48 & \multirow{3}{*}{$\begin{array}{l}\text { Cohort from University } \\
\text { of Washington/Group } \\
\text { Health Cooperative } \\
\text { Alzheimer's Disease } \\
\text { Patient Registry, USA }\end{array}$} & AD: CERAD, Braak stages > IV & \multirow[t]{3}{*}{ All } & AD: NINCDS/ADRDA (1984) \\
\hline & $\begin{array}{l}\mathrm{AD} / \mathrm{LBP} \\
65\end{array}$ & & $\begin{array}{l}\text { LB/AD: AD + synuclein (amygdala, } \\
\text { SN) }\end{array}$ & & \multirow{2}{*}{$\begin{array}{l}\text { DLB: missing criteria because study was } \\
\text { started before the consensus criteria for DLB } \\
\text { was established. }\end{array}$} \\
\hline & LBP 22 & & $\begin{array}{l}\text { LB: Braak stages < III, synuclein } \\
\text { (amygdala, SN) }\end{array}$ & & \\
\hline \multirow{5}{*}{$\begin{array}{l}\text { Stavitsky et al., } \\
2006 \text { [19] }\end{array}$} & AD 55 & \multirow{2}{*}{$\begin{array}{l}\text { Cohort of the Predictors } \\
\text { Study, 1997: }\end{array}$} & AD: CERAD & \multirow[t]{5}{*}{12} & AD: NINCDS/ADRDA (1984) \\
\hline & \multirow[t]{4}{*}{ DLB 28} & & \multirow{4}{*}{$\begin{array}{l}\text { LB: semi quantitative ubiquitin } \\
\text { (hippocampus, cingulate gyrus, } \\
\text { insula cortex) }\end{array}$} & & \multirow{4}{*}{ DLB: consensus criteria (1996) } \\
\hline & & Columbia University & & & \\
\hline & & $\begin{array}{l}\text { Johns Hopkins } \\
\text { University, }\end{array}$ & & & \\
\hline & & $\begin{array}{l}\text { Massachusetts General } \\
\text { Hospital, USA }\end{array}$ & & & \\
\hline \multirow{3}{*}{$\begin{array}{l}\text { Williams et al., } \\
2006 \text { [27] }\end{array}$} & AD 252 & \multirow{3}{*}{$\begin{array}{l}\text { Cohort from } \\
\text { Washington University, } \\
\text { USA }\end{array}$} & \multirow{2}{*}{$\begin{array}{l}\text { AD: NIA-RI quantification of diffuse } \\
\text { and neuritic depositions in } 10 \text { cor- } \\
\text { tical regions }\end{array}$} & \multirow[t]{3}{*}{ All } & AD: NINCDS/ADRDA (1984) \\
\hline & \multirow[t]{2}{*}{ DLB 63} & & & & \multirow[t]{2}{*}{ DLB: consensus criteria (1996) } \\
\hline & & & LB: synuclein & & \\
\hline \multirow{3}{*}{$\begin{array}{l}\text { Hamilton et al., } \\
2008[28]\end{array}$} & $\mathrm{AD} 44$ & \multirow{3}{*}{$\begin{array}{l}\text { University of California, } \\
\text { Alzheimer's disease } \\
\text { center San Diego, } 1985 \\
\text { to 2002, USA }\end{array}$} & AD: modified Braak staging, NIA-RI & \multirow[t]{3}{*}{ All } & AD: NIA-RI and CERAD (1988) \\
\hline & \multirow{2}{*}{ DLB 22} & & (1997) and & & \multirow{2}{*}{ DLB: consensus criteria (1996) } \\
\hline & & & $\begin{array}{l}\text { LB: H + E, ubiquitin (1996) synuclein } \\
\text { (2005) }\end{array}$ & & \\
\hline \multirow{2}{*}{$\begin{array}{l}\text { Hanyu et al., } \\
2009 \text { [29] }\end{array}$} & AD 111 & \multirow{2}{*}{$\begin{array}{l}\text { Memory Clinic of Tokyo } \\
\text { Medical University, } 2000 \\
\text { to 2006, Japan }\end{array}$} & & \multirow[t]{2}{*}{0} & AD: NINCDS/ADRDA (1984) \\
\hline & DLB 56 & & & & DLB: consensus criteria (1996) \\
\hline \multirow[t]{3}{*}{$\begin{array}{l}\text { Nelson et al., } \\
2009 \text { [16] }\end{array}$} & AD 107 & $\begin{array}{l}\text { National Alzheimer's } \\
\text { Coordinating Center } \\
\text { (NACC) Registry - } 31 \text { AD } \\
\text { centers in USA, }\end{array}$ & AD: NIA-RI & All & AD: CERAD (1988) \\
\hline & $\begin{array}{l}\mathrm{AD} / \mathrm{DLB} \\
27\end{array}$ & \multirow{2}{*}{$\begin{array}{l}\text { University of Kentucky } \\
\text { Alzheimer's Disease } \\
\text { Center, USA }\end{array}$} & \multirow[t]{2}{*}{ LB: Braak staging and CERAD } & & \multirow[t]{2}{*}{ DLB: consensus criteria (1996) } \\
\hline & DLB 9 & & & & \\
\hline \multirow{2}{*}{$\begin{array}{l}\text { Wood et al., } \\
2012 \text { [30] }\end{array}$} & AD 16 & \multirow[t]{2}{*}{ Newcastle University, UK } & & 0 & AD: NINCDS/ADRDA (1984) \\
\hline & DLB 12 & & & & DLB: consensus criteria (2005) or (1996) \\
\hline \multirow{2}{*}{$\begin{array}{l}\text { Walker et al. } \\
2012 \text { [31] }\end{array}$} & AD 100 & \multirow[t]{2}{*}{40 European sites } & \multirow[t]{2}{*}{${ }^{123}$ |-FTP-SPECT as verifying method } & \multirow[t]{2}{*}{0} & AD: NINCDS/ADRDA (1984) \\
\hline & DLB 58 & & & & DLB: consensus criteria (1996) \\
\hline
\end{tabular}

AD, Alzheimer's disease; ADRC, Alzheimer's Disease Research Center; CERAD, The Consortium to Establish a Registry for Alzheimer's Disease; CFV, creasyl fast violet; DLB, dementia with Lewy bodies; $\mathrm{H}+\mathrm{E}$, hematoxylin and eosin staining; I-FTP-SPECT, ioflupane single-photon emission computed tomography; LB Lewy body; LBV, Lewy body variant; LBP, Lewy body pathology; NIA-RI, National Institute on Aging-Reagan; NINCDS/ADRDA, National Institute of Neurological and Communication Disorders and Stroke/Alzheimer's Disease and Related Disorders Association; SDLT, senile dementia of Lewy body type; SN, substantia nigra.

20 years. In studies with short follow-up periods, the MMSE may not be a reliable measure, as Clark, Sheppard, Fillenbaum et al. (1999) [36] have argued that MMSE registrations need to be separated by at least three years in order to be a reliable measure of cognitive decline in AD.

Only few studies investigated, or reported, subgroups with different cognitive profiles in DLB. It could be due to a low number of cases in several studies, and subsequent low statistical power. People die from dementia or reach an endpoint where they are not capable of performing cognitive tests, and therefore in several studies there was a lower number of patients towards the end of the study. This is challenging when performing statistical analysis. Our search did not cover the issue of subgroups with different cognitive profiles thoroughly, as we only included studies comparing DLB with AD, and not studies describing cognitive decline in DLB and potential subgroups alone. However, there are some data that support the hypothesis that there are subgroups in DLB with different cognitive profiles, and subgroups with poor initial visuospatial function may have a more rapid decline than DLB with good visuospatial function [28].

Due to overlapping symptoms, it can be difficult to determine the correct diagnosis ante mortem between the pure form of $\mathrm{AD}$, mixed $\mathrm{AD} / \mathrm{DLB}$ and the pure form of DLB. Because clinical criteria cannot distinguish with certainty the individual pathology, the gold standard for validating the clinical assessment is neuropathological diagnosis. Clinical criteria may have a low sensitivity in 
particular for DLB, which could have been a source of bias in studies that did not include a neuropathological validation of the diagnosis. However, dementia is a clinical diagnosis and both AD and DLB pathology can be found also in cognitively normal elderly subjects. In one study with autopsy, $50 \%$ of cases with widespread $\alpha$ synucleinopathy did not show any clinical signs of dementia [37].

In most studies with autopsy, consensus neuropathological criteria were used. Even though not all included studies used consistent and the same neuropathological methods and criteria, and many also used varying combinations, use of post-mortem verification at least increases the validity of the clinical diagnosis.

It is also important to mention that the sensitivity for detecting Lewy bodies has increased with anti-ubiquitin immunostaining, where tau-positive samples indicate Alzheimer's pathology. Anti- $\alpha$-synuclein immunostaining has been incorporated in the assessment, which is most sensitive for Lewy body pathology [2]. Thus, the neuropathological identification of cases may have been less accurate before the new methods were established, and more reliable staging strategies have been developed [38].

A complicating issue is the frequent occurrence of mixed pathology [39], and to underline the complexity of dementia and its pathology, at least four distinct pathological phenotypes have been identified between AD and DLB [40]. According to Schneider et al. (2012) [7], the locus of neuropathology is associated with a faster decline in cognition. A neocortical type of Lewy body pathology is associated with increased odds of dementia and a faster decline in episodic, semantic and working memory. The limbic-type is more associated with more rapid decline in visuospatial function. Olichney et al. (1998) [3], concluded that patients with Lewy body variant decline faster than patients with Alzheimer's disease. This statement has often been used with reference to rapid progression in DLB, but it actually refers to an AD variant with Lewy body pathology, not to pure DLB. It should be emphasized that it is still uncertain whether $\mathrm{AD}$ and $\mathrm{DLB}$ are two independent pathologies that may coexist, or the pathologies are related, or one of them is a consequence of the other.

\section{Conclusion}

Only 6 of the 18 included studies in this review found some differences in cognitive decline between DLB and $\mathrm{AD}$ over time, and only one of them found a faster decline in DLB. It is difficult to draw firm conclusions based on available studies, since the results are contradictory. Future studies will need to apply recent diagnostic criteria, as well as extensive diagnostic evaluation and autopsy to confirm the diagnosis. Studies with large enough samples, adapted cognitive tests, more than one year of follow up and multivariate statistical analysis are also needed. Inclusion of mild cognitive impairment patients, with subclinical manifestations and an increased risk of developing DLB (for example, who present rapid eye-movement (REM) sleep behavior disorder) could also strengthen the studies. Our final conclusion is that the studies in this review support neither the hypothesis of a faster cognitive decline in DLB, nor in AD.

\section{Abbreviations}

AD: Alzheimer's disease; ADL: activities of daily living; CAMCOG: Cambride cognitive examination; CDT: clock drawing test; CERAD: Consortium to Establish a Registry for Alzheimer's Disease evaluation; DLB: dementia with Lewy bodies; DRS: dementia rating scale; HVLT-R: Hopkins verbal learning test-revised; MMSE: mini mental state examination; NEVIP: Newcastle visual perception battery; NINCDS/ADRDA: National Institute of Neurological and Communication Disorders and Stroke/Alzheimer's Disease and Related Disorders Association; SPECT: ioflupane single-photon emission computed tomotgraphy; WISC-R: Wechsler intelligence scale for children-revised.

\section{Competing interests}

Dag Aarsland has received research support and honoraria from $\mathrm{H}$ Lundbeck, Novartis Pharmaceuticals and GE Health. None of the other authors have competing interests.

\section{Authors' contributions}

MHB and LC have made the conception and design, data acquisition, analysis and interpretation of data, and drafted the manuscript. AR, MJH, CJ, $\mathrm{KB}$ and $\mathrm{DA}$ have contributed to the analysis and interpretation of data, and revised the manuscript critically for important intellectual content. KB also performed the meta-analysis. All authors have read and approved the final version of the manuscript.

\section{Acknowledgement}

We want to thank the librarian in Helse Fonna, Tonje Velde, for helping us with the systematic literature search.

\section{Author details}

'Section of Mental Health Research, Clinic of Psychiatry, Helse-Fonna HF Haugesund Hospital, Post-box 2170, 5504 Haugesund, Norway. ${ }^{2}$ Old Age Department, Clinic of Psychiatry, Helse-Fonna HF Haugesund Hospital, Post-box 2170, 5504 Haugesund, Norway. ${ }^{3}$ Neurological Department, Clinic of Medicine, Helse-Fonna HF Haugesund Hospital, Post-box 2170, 5504 Haugesund, Norway. ${ }^{4}$ Department of Clinical Psychology, University of Bergen, Christies gate 12, 5015 Bergen, Norway. ${ }^{5}$ NKS Olaviken Hospital for Old Age Psychiatry, Ulriksdal 8, 5009 Bergen, Norway. ${ }^{6}$ Faculty of Medicine, University of Bergen, Post-box 78045020 Bergen, Norway. ${ }^{7}$ TIPS, Regional Centre for Clinical Research in Psychosis, Stavanger University Hospital, Post-box 8100, 4068 Stavanger, Norway. ${ }^{8}$ Network for Medical Sciences, Stavanger University Hospital, Post-box 8100, 4068 Stavanger, Norway. ${ }^{9}$ Centre for Age Related Medicine, Stavanger University Hospital, Post-box 8100, 4068 Stavanger, Norway. ${ }^{10}$ Department NVS, Center for Alzheimer Research, Division for Neurogeriatrics, Karolinska Institutet, 14157 Huddinge, Sweden.

Received: 15 January 2014 Accepted: 25 July 2014

Published online: 16 September 2014

\section{References}

1. Aarsland D, Rongve A, Piepenstock Nore S, Skogseth R, Skulstad S, Ehrt U, Hoprekstad D, Ballard C: Frequency and Case Identification of Dementia with Lewy Bodies Using the Revised Consensus Criteria. Dement Geriatr Cogn Disord 2008, 26:445-452.

2. McKeith IGI, Dickson DWD, Lowe JJ, Emre MM, O. apos Brien JTJ, Feldman $\mathrm{HH}$, Cummings JJ, Duda JEJ, Lippa CC, Perry EKE, Aarsland DD, Arai HH, Ballard CGC, Boeve BB, Burn DJD, Costa DD, Del Ser T, Dubois BB, Galasko DD, Gauthier SS, Goetz CGC, Gomez-Tortosa EE, Halliday GG, Hansen LAL, Hardy JJ, Iwatsubo TT, Kalaria RNR, Kaufer DD, Kenny RAR, Korczyn AA, et al: 
Diagnosis and management of dementia with Lewy bodies: third report of the DLB Consortium. Neurology 2005, 65:1863-1872.

3. Olichney JM, Galasko D, Salmon DP, Hofstetter CR, Hansen LA, Katzman R, Thal $\mathrm{L}$ : Cognitive decline is faster in Lewy body variant than in Alzheimer's disease. Neurology 1998, 51:351-357.

4. Oesterhus R, Soennesyn H, Rongve A, Ballard C, Aarsland D, Vossius C: Long-term mortality in a cohort of home-dwelling elderly with mild Alzheimer's disease and Lewy body dementia. Dement Geriatr Cogn Disord 2014, 8:161-169.

5. Rongve A, Vossius C, Nore S, Testad I, Aarsland D: Time until nursing home admission in people with mild dementia: comparison of dementia with Lewy bodies and Alzheimer's dementia. Int I Geriat Psychiatry 2014, 29:392-398.

6. Vossius C, Rongve A, Testad I, Wimo A, Aarsland D: The use and costs of formal care in newly diagnosed dementia: a three-year prospective follow-up study. Am J Geriatr Psychiatry 2014, 22:381-388.

7. Schneider JA, Arvanitakis Z, Yu L, Boyle PA, Leurgans SE, Bennett DA: Cognitive impairment, decline and fluctuations in older communitydwelling subjects with Lewy bodies. Brain 2012, 135:3005-3014.

8. Lippa CF, Duda JE, Grossman M, Hurtig HI, Aarsland D, Boeve BF, Brooks DJ, Dickson DW, Dubois B, Emre M, Fahn S, Farmer JM, Galasko D, Galvin JE, Goetz CG, Growdon JH, Gwinn-Hardy KA, Hardy J, Heutink P, Iwatsubo T, Kosaka K, Lee VM-Y, Leverenz JB, Masliah E, McKeith IG, Nussbaum RL, Olanow CW, Ravina BM, Singleton AB, Tanner CM, et al: DLB and PDD boundary issues: diagnosis, treatment, molecular pathology, and biomarkers. Neurology 2007, 68:812-819.

9. Janvin CC, Larsen JP, Aarsland D, Hugdahl K: Subtypes of mild cognitive impairment in Parkinson's disease: progression to dementia. Mov Disord 2006, 21:1343-1349.

10. Williams-Gray CH, Foltynie T, Brayne CEG, Robbins TW, Barker RA: Evolution of cognitive dysfunction in an incident Parkinson's disease cohort. Brain 2007, 130:1787-1798.

11. Clinton LK, Blurton-Jones M, Myczek K, Trojanowski JQ, LaFerla FM: Synergistic Interactions between Abeta, tau, and a-synuclein: acceleration of neuropathology and cognitive decline. J Neurosci 2010, 30:7281-7289.

12. Aarsland D, Zaccai J, Brayne C: A systematic review of prevalence studies of dementia in Parkinson's disease. Mov Disord 2005, 20:1255-1263.

13. Borenstein M, Hedges LV, Higgins JPT, Rothstein HR: Introduction to Meta-Analysis. New Jersey: Wiley; 2011.

14. Higgins JP, Thompson SG, Deeks JJ, Altman DG: Measuring inconsistency in meta-analyses. BMJ 2003, 327:557-560.

15. Kraybill ML, Larson EB, Tsuang DW, Teri L, McCormick WC, Bowen JD, Kukull WA Leverenz JB, Cherrier MM: Cognitive differences in dementia patients with autopsy-verified AD, Lewy body pathology, or both. Neurology 2005, 64:2069-2073.

16. Nelson PT, Kryscio RJ, Jicha GA, Abner EL, Schmitt FA, Xu LO, Cooper G, Smith CD, Markesbery WR: Relative preservation of MMSE scores in autopsy-proven dementia with Lewy bodies. Neurology 2009, 73:1127-1133.

17. Ballard C, Patel A, Oyebode F, Wilcock G: Cognitive decline in patients with Alzheimer's disease, vascular dementia and senile dementia of Lewy body type. Age Ageing 1996, 25:209-213.

18. Heyman A, Fillenbaum GG, Gearing M, Mirra SS, Welsh-Bohmer KA, Peterson B, Pieper C: Comparison of Lewy body variant of Alzheimer's disease with pure Alzheimer's disease: Consortium to Establish a Registry for Alzheimer's Disease, Part XIX. Neurology 1999, 52:1839-1844.

19. Stavitsky K, Brickman AM, Scarmeas N, Torgan RL, Tang M-X, Albert M, Brandt J, Blacker D, Stern Y: The progression of cognition, psychiatric symptoms, and functional abilities in dementia with Lewy bodies and Alzheimer disease. Arch Neurol 2006, 63:1450-1456.

20. McKeith IG, Perry RH, Fairbarin AF, Jabeen S, Perry EK: Operational criteria for senile dementia for Lewy body type (SDLT). Psychol Med 1992, 22:911-922.

21. Ballard CG, O'Brien J, Lowery K, Ayre GA, Harrison R, Perry R, Ince P, Neill D, McKeith IG: A prospective study of dementia with Lewy Bodies. Age Ageing 1998, 27:631-636

22. Lopez OL, Wisniewski S, Hamilton RL, Becker JT, Kaufer DI, DeKosky ST: Predictors of progression in patients with $A D$ and Lewy bodies. Neurology 2000, 54:1774-1779.

23. Stern Y, Jacobs D, Goldman J, Gomez-Tortosa E, Hyman BT, Liu Y, Troncoso J, Marder K, Tang MX, Brandt J, Albert M: An investigation of clinical correlates of Lewy bodies in autopsy-proven Alzheimer disease. Arch Neurol 2001, 58:460-465.

24. Ballard C, O'Brien J, Morris CM, Barber R, Swann A, Neill D, McKeith I: The progression of cognitive impairment in dementia with Lewy bodies, vascular dementia and Alzheimer's disease. Int J Geriatri Psychiatry 2001, 16:499-503.

25. Helmes E, Bowler J, Merskey H, Munoz DG, Hachinski VC: Rates of cognitive decline in Alzheimer's disease and dementia with Lewy bodies. Dement Geriatr Cogn Disord 2003, 15:67-71.

26. Johnson DK, Morris JC, Galvin JE: Verbal and visuospatial deficits in dementia with Lewy bodies. Neurology 2005, 65:1232-1238.

27. Williams MM, Xiong C, Morris JC, Galvin JE: Survival and mortality differences between dementia with Lewy bodies vs Alzheimer disease. Neurology 2006, 67:1935-1941.

28. Hamilton JM, Salmon DP, Galasko D, Raman R, Emond J, Hansen LA, Masliah E, Thal $\mathrm{L}$ : Visuospatial deficits predict rate of cognitive decline in autopsy-verified dementia with Lewy bodies. Neuropsychology 2008, 22:729-737.

29. Hanyu H, Sato T, Hiraro K, Kanetaka H, Sakurai H, Iwamoto T: Differences in clinical course between dementia with Lewy bodies and Alzheimer's disease. Eur J Neurol 2009, 16:212-217.

30. Wood JS, Watson R, Firbank MJ, Mosimann UP, Barber R, Blamire AM, O'Brien JT: Longitudinal testing of visual perception in dementia with Lewy bodies and Alzheimer's disease. Int J Geriat Psychiatry 2013, 28:567-572.

31. Walker Z, McKeith I, Rodda J, Qassem T, Tatsch K, Booij J, Darcourt J, O'Brien J: Comparison of cognitive decline between dementia with Lewy bodies and Alzheimer's disease: a cohort study. BMJ Open 2012, 2:e000380.

32. Metzler-Baddeley C: A review of cognitive impairments in dementia with Lewy bodies relative to Alzheimer's disease and Parkinson's disease with dementia. Cortex 2007, 43:583-600

33. Brown J, Pengas G, Dawson K, Brown LA, Clatworthy P: Self administered cognitive screening test (TYM) for detection of Alzheimer's disease: cross sectional study. BMJ 2009, 338:b2030.

34. Yoshizawa H, Vonsattel JPG, Honig LS: Early neuropsychological discriminants for Lewy body disease: an autopsy series. J Neurol Neurosurg Psychiatr 2013, 84:1326-1330.

35. Galasko DR, Gould RL, Abramson IS, Salmon DP: Measuring cognitive change in a cohort of patients with Alzheimer's disease. Stat Med 2000, 19:1421-1432.

36. Clark CM, Sheppard L, Fillenbaum GG, Galasko D, Morris JC, Koss E, Mohs R Heyman A: Variability in annual Mini-Mental State Examination score in patients with probable Alzheimer disease: a clinical perspective of data from the Consortium to Establish a Registry for Alzheimer's Disease. Arch Neurol 1999, 56:857-862.

37. Parkkinen L, Pirttilä T, Alafuzoff I: Applicability of current staging/ categorization of a-synuclein pathology and their clinical relevance. Acta Neuropathol 2008, 115:399-407.

38. Alafuzoff I, Ince PG, Arzberger T, Al-Sarraj S, Bell J, Bodi I, Bogdanovic N, Bugiani O, Ferrer I, Gelpi E, Gentleman S, Giaccone G, Ironside JW, Kavantzas N, King A, Korkolopoulou P, Kovács GG, Meyronet D, Monoranu C, Parchi P, Parkkinen L, Patsouris E, Roggendorf W, Rozemuller A, Stadelmann-Nessler C, Streichenberger N, Thal DR, Kretzschmar H: Staging/typing of Lewy body related a-synuclein pathology: a study of the BrainNet Europe Consortium. Acta Neuropathol 2009, 117:635-652

39. Schneider JA, Arvanitakis Z, Bang W, Bennett DA: Mixed brain pathologies account for most dementia cases in community-dwelling older persons. Neurology 2007, 69:2197-2204.

40. McCann H, Stevens $\mathrm{CH}$, Cartwright $\mathrm{H}$, Halliday GM: a-Synucleinopathy phenotypes. Parkinsonism Relat Disord 2014, 20:62-67.

\section{doi:10.1186/s13195-014-0053-6}

Cite this article as: Breitve et al:: A systematic review of cognitive decline in dementia with Lewy bodies versus Alzheimer's disease. Alzheimer's Research \& Therapy 2014 6:53. 